\title{
Inevitability of the Pain Experience in Margaret Mazzantini's Novels
}

\author{
Nikica Mihaljević \\ Mira Petrović \\ Dipartimento di Italianistica \\ Università degli studi di Spalato, Croazia \\ nikica@ffst.hr
}

\section{Doi:10.5901/ajis.2013.v2n8p511}

\section{Abstract}

In the paper we focus on the motif of pain in a literary text with the aim to analyze the importance of suffering in the lives of characters and to find out whether the pain determines the behaviour and the reactions of characters. We start from the assumption that the experience of pain influences the characters in the way that it incites them to re-examine their decisions and that it encourages them to transgress the boundaries in certain situations. In the analysis we study two novels by the contemporary Italian authoress Margaret Mazzantini, in which the individual suffering is intertwined with the suffering of the society; namely, the pain of the motherhood, whether unrealized one or the one in which a mother is not able to protect her own child, is entwined with the suffering of the victims of war or those forced to exile. The pain is, therefore, analyzed on two levels: that of the characters and that of the setting. The suffering of the individual transforms into the prism which helps us to understand the collective suffering, as well as the responsibility of the individual and the collective responsibility. In this way we find out the moral value of the pain since what results in the transition from the individual to collective suffering is the bond that unites the people in the common experience of pain.

Keywords: pain, suffering, motherhood, war, body, Italian literature.

\section{Introduzione}

In Storia del nulla (Givone 2011), analizzando in primo luogo il nulla nella storia della filosofia, Sergio Givone affronta anche il concetto del dolore ${ }^{1}$ da vari punti di vista, problematizzandolo attraverso le tesi di diversi filosofi e scrittori attraverso i secoli. In ciò vi sono, come si può supporre, delle tesi contrastanti, come, ad esempio, quella che troviamo in Eschilo e in Nietzsche, ovvero la questione dell'ineluttabilità del dolore. Givone cita Severino quando interpreta Eschilo, e spiega che per quest'ultimo il dolore rappresenta un peso vano per l'animo, e l'aspetto principale di quest'esperienza è che la liberazione dal dolore avvenga nella verità. (Givone 2011:28-29) In Eschilo, dunque, troviamo, in nome della verità, una rinuncia alla vita in quanto dolente, mentre per Nietzsche, invece, il dolore è connaturato alla vita, fa tutt'uno con essa, e quindi non ha senso cercare di liberarsene. (Givone 2011:28-29) Interpretando, invece, il pensiero di Zeus, collegata con la giustizia o l'ingiustizia assegnata a Prometeo, il dolore diventa "organo della conoscenza" ovvero non vi è "nessuna conoscenza se non attraverso il dolore". (Givone 2011:23-32) Riassumendo, l'uomo non può rinunciare né alla conoscenza né al dolore poiché "la conoscenza è fedeltà al dolore."(Givone 2011:32)2

Nella problematica del dolore due sono gli aspetti fondamentali ed indissolubili insiti in questo concetto: il dolore è

\footnotetext{
${ }^{1}$ Secondo Givone, nello Zibaldone di Leopardi viene esposta una metafisica del nulla in quanto la storia del nulla è collegata anche con la storia del dolore in quanto tutto le cose, e Dio stesso, hanno origine dal nulla, e, quindi, anche il dolore è un nulla. (Cfr. Givone 2011:142.) "Tutto è nulla al mondo, anche la mia disperazione, della quale ogni uomo anche savi, ma più tranquillo, ed io stesso certamente in un'ora più quieta conoscerò la vanità e l'irragionevolezza e l'immaginario. Misero me, è vano, è un nulla anche questo mio dolore, che in un certo tempo passerà e s'annullerà, lasciandomi in un vôto universale $\mathrm{e}$ in un'indolenza terribile che mi farà incapace anche di dolermi." (Leopardi 1983:71).

${ }^{2}$ Luigi Pareyson rileva che ciò che l'uomo avvicina il suo essere a Dio soltanto attraverso la sofferenza, arrivando così ad una "consofferenza divina e umana": "Ė estremamente tragico che solo nel dolore Dio riesca a soccorrere l'uomo e l'uomo giunga a redimersi. Ma è proprio in questa con sofferenza divina e umana che il dolore si rivela come l'unica forza che riesce ad avere ragione del male. Questo principio è uno dei capisaldi del pensiero tragico: che fra l'uomo e Dio non ci sia collaborazione nella grazia se prima non c'è stata nella sofferenza [...]." (Pareyson 1989:33).
} 
un'esperienza esclusivamente individuale ed è collegato strettamente con la sofferenza dell'individuo. ${ }^{3}$ Di conseguenza, dato che il dolore è un'esperienza profondamente radicata nell'uomo e nel mondo, tale argomento appare molto frequentemente nell'ambito letterario. ${ }^{4}$

II dolore è un'esperienza del tutto personale, soltanto in parte comunicabile, che nasce con l'uomo trasformandosi allo stesso tempo in una condizione inseparabile della sua vita. Nonostante sia un'esperienza comune a tutti gli uomini, non è possibile delineare con precisione le sensazioni che essa procura, dato che la capacità di raccontare il dolore in parole è legata a varie esperienze precedenti al momento della sofferenza, ognuna di esse diverse nella loro singolarità. ${ }^{5}$ Condividendo l'esperienza della sofferenza con altre persone, essa sembra più facile da sopportare, come se si alleggerisse il suo peso, e a chi ne soffre sembra pare che rimanga solo una parte visto che pare che gli altri, con i quali è condivisa, abbiano altre sue parti. ${ }^{6}$ Essendo un'esperienza individuale, il dolore è anche strettamente legato al corpo in cui viene provocata questa sofferenza.

Strettamente legati al corpo sono anche i processi fisiologici come la mestruazione ${ }^{7}$ che caratterizza esclusivamente il corpo femminile e, conseguentemente, attraverso questa particolarità la donna viene definita. (Cfr. Carson 2006:94) II ciclo mestruale appare ripugnante all'uomo, e non per la mancanza della pulizia o della salute, come spiega Kristeva nei Poteri dell'orrore, quanto perché rappresentano la forza indistruttibile della donna in quanto esclusivamente femminile ed inconcepibile nel pensiero maschile. Proprio questa caratteristica rende la donna superiore all'uomo, per cui sembra che una tale forza disturbi l'identità stabilita tra i sessi, nonché il sistema, l'ordine, le regole, ed i confini tra l'interno e l'esterno. ${ }^{8}$

Il legame stretto tra il corpo e la sofferenza viene focalizzato da Franco Rella il quale rileva che non solo la sofferenza fisica ma anche quella interiore, cioè emotiva o mentale, è visibile dall'esamina del corpo dell'individuo che soffre. II corpo in questo senso diventa spesso lo strumento di comunicazione per la sofferenza: "Ma il corpo si scopre, si denuda, si offre anche nella sofferenza, anche in quella dimensione estrema della sofferenza che è la vecchiaia. La sofferenza, si è detto, inchioda l'io al corpo: ne fa un non-oltre. [...] Eppure, anche nella sofferenza, come nell'eros, c'è un punto di sporgenza verso altro, oltre i confini della sofferenza stessa." (Rella 2012b:22) Ciò si rivela un fatto importante nei romanzi della Mazzantini, e quindi nell'analisi viene focalizzato in primo luogo il corpo, come mezzo ed anche espressione ultima di esternazione del dolore, dato che il dolore, come sottolineato da Rafael Argullol, "possiede anche l'incredibile virtù di far sentire, con un'acutezza staordinaria, il proprio corpo" (Argullol 2010:11)

${ }^{3}$ Salvatore Natoli mette in rilievo che il dolore si manifesta come la voce del nulla, la potenza negativa da cui è affetto ogni essere. (Cfr. Natoli 2002:42)

${ }^{4}$ Dostoevskii, ad esempio, nelle Memorie del sottosuolo afferma che l'uomo ama la sofferenza, perfino a tal punto che egli non rinuncerà mai alla sofferenza autentica, cioè alla distruzione e al caos: "Non potrebbe darsi che all'uomo non piaccia soltanto la prosperità? Non potrebbe darsi che gli piaccia altrettanto la sofferenza? Non può darsi che la sofferenza sia per lui vantaggiosa esattamente nella stessa misura della prosperità? E all'uomo talvolta piace terribilmente la sofferenza, gli piace alla follia. [...] Sarà bene 0 sarà male, ma talvolta distruggere qualcosa può anche essere molto piacevole". (Dostoevskij 1989:50). Nei Fratelli Karamazov, d'altra parte, Dostoevskij "fa della condizione umana di sofferenza e di dolore un problema metafisico che si proietta sull'essere stesso di Dio". (Dostoevskij 1989:44.). Per Leopardi, invece, il dolore non è il sentimento più greve che l'uomo possa sperimentare: "Il dolore o la disperazione che nasce dalle grandi passioni e illusioni o da qualunque sventura della vita, non è paragonabile all'affogamento che nasce dalla certezza e dal sentimento vivo della nullità di tutte le cose, e della impossibilità di esser felice a questo mondo, e dalla immensità del vuoto che si sente nell'anima." (Leopardi 1983:115).

${ }^{5}$ Felice Eugenio Agrò spiega il significato del dolore con le seguenti parole: "Il dolore è un'esperienza somato-psichica caratterizzata da connotati biologici, affettivi, relazionali, spirituali, culturali non separabili tra loro. Ogni individuo impara ad esprimere il dolore con parole che sono il frutto di vissuti psicologici legati a esperienze traumatiche precedenti, soprattutto della propria infanzia ed adolescenza. Pur essendo, il dolore, un'esperienza cui partecipano tutti gli uomini, è difficile darne una definizione esauriente, e, ancor meno, riuscire ad elencare tutti i diversi tipi del dolore che ciascuno degli individui ha sperimentato nella sua vita [...]." (Agrò 2003:17).

${ }^{6} \mathrm{~A}$ proposito del metodo per alleviare la sofferenza, Bonelli rileva: "Con ciò va detto che attraverso un portare e sopportare il dolore comune, insieme e l'uno per l'altro, nel quale uno è per così dire unito al dolore dell'altro attraverso una partecipazione amorevole e soprattutto attraverso un'attiva misericordia, per colui che soffre può essere essenzialmente alleggerita l'amarezza della sua malattia." (Bonelli 2003:73).

7 "Certamente le escrezioni, l'orina, la merda, e pure lo sperma e le mestruazioni, anche se queste sono più immediatamente legate alla sfera sessuale, provengono da dentro il corpo, rinviano a una sua dimensione celata e non immediatamente visibile. [...] Rinviano, secondo Joyce, più propriamente alla dimensione profonda della vita, essa stessa, come apollinea, generatrici di immagini e di pensiero." (Rella 2012:160).

${ }^{8}$ Cfr. Kristeva 1989:10. In quest'opera Kristeva cerca di analizzare e di spiegare questa materialità del corpo femminile, ossia le sue pulsazioni ed emanazioni viste come ripugnanti nella cultura che vuole separare la razionalità e l'intelletto dalla corporalità. 
In quest'analisi si cerca di dimostrare come l'inevitabilità del dolore nella vita dei protagonisti influenzi le loro decisioni e cambi il loro destino, perché il dolore mette in moto l'azione e costringe i personaggi al cambiamento, alla ribellione, alla responsabilità. A volte il dolore provoca la disperazione, spingendo i personaggi a combattere uno contro l'altro, e talvolta li costringe al silenzio, alla paura e all'impossibilità di portare avanti la vita. Ciò diventa evidente soprattutto nei casi in cui i personaggi sono confrontati con la violenza, con l'odio, con la solitudine e con l'esclusione.

\section{L'inevitabilità del dolore}

I tipi del dolore che si possono individuare nell'opera della Mazzantini sono il dolore fisico o la malattia, il dolore psichico e morale, nonché il dolore provocato dalla violenza.

II Discorso sull'indole del piacere e del dolore di Pietro Verri si apre con l'affermazione che le azioni umane sono tutte determinate 0 dal piacere 0 dal dolore: "La sensibilità dell'uomo, il grande arcano, al quale è stata ridotta come a generale principio ogni azione della fisica sopra di noi, si divide e scompone in due elementi, e sono amor del piacere e fuga del dolore: tale almeno è la comune opinione degli uomini pensatori e maestri." (Verri 1964:3) Secondo Verri, quindi, ogni atteggiamento umano è la conseguenza dell'esperienza o del dolore o del piacere, i quali danno vita l'uno all'altro reciprocamente. ${ }^{9}$ Eppure, nonostante il dolore rappresenti un elemento facente parte dell'esistenza umana, esso è, tuttavia, difficilmente accettabile, in quanto si rivela un'esperienza radicale, la quale riconduce alla percezione progressiva della riduzione della possibilità di vita, quindi, della morte. Conseguentemente, l'esperienza della malattia, ovvero del momento in cui il corpo soffre, dimostra quanto precario sia quello che si ha e quello che si è. (Cfr. Natoli 2002:34).

L'apparire del dolore, quindi, determina un cambiamento di prospettiva che porta il malato ad interrogarsi su se stesso, ma anche sul rapporto con il mondo e con altre persone. (Russo 2003:99-116.)

In primo luogo, la malattia si può capire come un atto di autorealizzazione che richiede l'autotrascendimento, il quale può portare all'esperienza religiosa, nonché ad una crescita ed apertura verso altre persone che ci circondano, sicché il dolore purifica la capacità e la necessità di amare. In questo modo il dolore influisce anche sulle relazioni della persona che soffre.

D'altra parte, il dolore rende l'uomo capace, in maggior misura, di attenzione e di apprensione verso se stesso ma anche verso il mondo. Quindi, sebbene il dolore causi nel malato un senso di solitudine e di esclusione dal mondo esterno ${ }^{10}$, esso può anche cambiare la percezione del mondo, cioè, grazie al dolore l'uomo riesce a capire l'ineluttabilità universale della sofferenza. Oltre a ciò, il dolore, pur essendo un fatto personale, è anche un evento cosmico, dato che la sofferenza non può essere individuale, come afferma Natoli, ma è una variazione del dolore universale, della crudeltà generale della vita. (Natoli 2002:29) Inoltre, "questo intreccio di singolare e di universale [...] permette a questa esperienza [del dolore] di farsi linguaggio" (Natoli 2002:8), cioè, grazie all'universalità del dolore, esso diventa comunicabile e si trasforma in un lutto possibile: del dolore si parla scambiando le esperienze per creare una più forte intimità e comprensione tra gli uomini. Ciò, del resto, contribuisce alla rappresentazione del dolore in arte, letteratura e filosofia.

Nel corso degli anni, il dolore diventa sempre più accentuato perché non esiste più alcuna certezza né base su cui appoggiare la propria esistenza. Esso avviene, secondo Alessandro Greco, perché non c'è più un modello di uomo al quale ispirarsi: l'uomo smette di essere il soggetto e diventa un fenomeno che si muove ed agisce in un vortice di molteplici attività senza regole, senza morale, senza religione, per cui diventa disorientato e la sua identità si spezza fino alla scomparsa dell'io. (Cfr. Greco 2004:17)

Quando si parla della sofferenza, un'importanza particolare va data al dolore causato dalla violenza. La violenza è strettamente legata alla problematica del male perché la sofferenza e il dolore sono l'espressione umana più tragica del male. Riguardo questo argomento, Rella mette in rilievo che in teologia, ad esempio, si rileva che il male è un defectum boni, una mancanza, una lacuna del bene. (Rella 2002:25) II male in sé non esiste, è solo una variazione negativa del bene. Dio, il sommo bene, ha dato all'uomo la libertà del bene e del male, trasformando così il mondo in un teatro della

\footnotetext{
${ }^{9}$ Secondo Verri, il dolore spinge l'uomo ad andare avanti, essendo la causa di tutti i movimenti umani, cioè "il bene nasce dal male, la sterilità produce l'abbondanza, la povertà fa nascere il coraggio, in una parola il dolore è il principio motore [...] dell'uomo, che senza di lui sarebbe un animale inerte e stupido, e perirebbe poco dopo di esser nato". (Verri 1964:23).

${ }^{10}$ Salvatore Natoli afferma che la solitudine e la sofferenza entrano l'una nell'altra al modo di un circolo vizioso, in quanto chi è colpito dal dolore si percepisce diverso e si esclude dal mondo, in quanto esso non può accettare la debolezza con cui il dolore viene associato. (Cfr. Natoli 2002:29)
} 
lotta continua per il bene e il male. (Rella 2002:25) Tuttavia, nel mondo in cui l'uomo diventa sempre di più distruttivo e violento, sembra che il bene si ritiri, che sia fuori dal mondo. Ciò ha la sua prova, secondo Rella, in Auschwitz, nell'incarnazione del male, in quanto in questi campi d'orrore il potere ha liberato e reso conoscibile la violenza e la volontà del male, le quali hanno dimostrato di essere una parte irriducibile dell'uomo e del mondo, ovvero il disumano che ha distrutto qualsiasi legge morale. (Rella 2002:50)

Nella prospettiva teologica, tuttavia, la sofferenza si rivela necessaria perché senza di essa non si potrebbe capire il bene e il male. Eppure, è forse facile accettare il fatto che, se il bene esiste, esiste anche il male come la sua contraddizione, in un rapporto reciproco; nella tragedia di Auschwitz, nell'orrore di ogni guerra, però, si può percepire solo l'indifferenza, l'impossibilità di distinguere il bene e il male, una coscienza vuota. In questo senso Rella spiega che, se si prende in considerazione la coscienza vuota degli uomini in guerra, essa diventa la coscienza proprio del defectum che è stato proposto dai teologi per chiarire l'inesistenza in sé del male, ma che, in realtà, lo afferma nella sua dimensione più lancinante: il vuoto e l'assenza entro cui il male può dilagare. (Rella 2002:27)

\section{L'espansione del dolore in un testo letterario:Venuto al mondo: il viaggio della speranza}

Il romanzo Venuto al mondo è una storia di amore e di guerra, del dolore di una madre e del dolore dell'Europa distrutta in un passato recente, ma è prima di tutto una storia di grandi perdite: della perdita d'identità, d'umanità, d'amore, di dignità, di una persona cara, e, a causa di questa perdita, la speranza di una vita migliore sembra impossibile.

Venuto al mondo è anche la storia di un viaggio, attraverso il quale i personaggi vengono confrontati con la paura, con i limiti dell'identità. II romanzo è la riflessione amara sulla vita, sul destino degli uomini, convinti di poter controllare il mondo, sull'amore che nasce dalla passione e cresce come il fiume, per ritrovare poi uno scoglio, un ostacolo insormontabile; sulla diffusione dell'odio, sull'assoluta indifferenza della guerra e del male che riduce tutto a niente.

La trama del romanzo si sposta in continuazione tra il presente (Gemma e Pietro a Sarajevo, con i ricordi della guerra) ed il passato (d'una parte, l'orrore e la paura della guerra, il dolore della città e degli abitanti di Sarajevo, e d'altra, la guerra personale ed intima di Gemma, nata dall'ossessione verso la maternità negata). Ciò porta alla superficie anche "lo scorrere inesorabile del tempo, la sua transitorietà" ${ }^{11}$, ma anche la possibilità della manipolazione di questa transitorietà in quanto, attraverso l'invocazione del passato, della memoria che conserva i ricordi dell'esistenza, la vita torna ad essere presente e sfida la morte. ${ }^{12} \|$ concetto della transitorietà pone anche al centro dell'attenzione il corpo e le sue trasformazioni, e sarà proprio il corpo di Gemma, e "il corpo" della città stessa, a dover affrontare i danni del tempo. ${ }^{13}$

All'inizio del romanzo Gemma viene delineata come un personaggio - ostaggio di un passato inquieto: "una donna invecchiata", "ferma nel tempo", che deve confrontarsi con il passato che ha provocato le conseguenze con le quali vive tutt'ora, "una ragazza sconfitta a un passo dalla vecchiaia", che deve trovare il coraggio per affrontare "quella voragine che nessuno ha mai chiuso". II viaggio diventa per lei un'espressione ritardata di una tristezza profonda, che non cerca una sua chiusura ma piuttosto una conferma della sua presenza. L'arrivo a Sarajevo è perciò come la riapertura di una ferita; si mette subito in rilievo il desiderio di Gemma di non essere dominata dall'orrore della città:

Non mi lascerò scoperchiare da questa città. Lascio passare le prime immagini senza registrarle veramente, brevi occhiate furtive, spezzoni, come francobolli bruciati. [...] Ho imparato che tutto può andare via, anche l'orrore può perdere le sue forme, stemperarsi in una nebulosa che lo altera, lo rende ridicolo, troppo assurdo per essere mai stato vero. (Mazzantini 2010:21)

Tuttavia, questa caratteristica di Gemma di non accettare la verità ("So spargere menzogne come pepite di verità"), nel corso del romanzo si dissolve pianamente, facendo immergere il lettore nel ricordo delle due battaglie che

${ }^{11}$ De Maglie, D. Dal Catino di zinco a Zorro: L'identità femminile in Margaret Mazzantini. http://www.altoadigecultura.org/pdf/r04_05.html (24.09.2011.) (15.05.2012.).

12 I ricordi consistono delle descrizioni della gente di Sarajevo che Gemma "mette a loro posto" nella sua memoria, cercando di dare il senso alla loro vita: "Che fine ha fatto [la cuoca]? Che fine fece il suo tegame carico di grasso, il suo golf di lana... il suo viso? Per me, quella donna è ancora lì, ferma all'angolo davanti al mercato di Bezistan, che ci sorride mentre ci sfama, e ci incoraggia a mangiare e a credere nel bene. A anche se una granata se l'è portata via, se un getto di fuoco ha sparso le sue povere cose, io giuro che è viva". (Mazzantini 2010:30).

${ }^{13}$ |l legame tra la transitorietà del tempo e la corporeità viene spiegato con queste parole da Franco Rella: "Dunque io sono perché il mio corpo si muove e occupa uno spazio, è soggetto al tempo e al mutamento. Leggo il mio movimento misurando la mia posizione in relazione a quella di altri corpi, percepisco il mio mutamento - e quindi il trascorrere del tempo - vedendo le trasformazioni di un volto che mi è stato prossimo, e che si decompone nel corso degli anni." (Rella 2012:34). 
hanno segnato la sua vita, quella per l'amore della sua vita e per il figlio che non arriva. II viaggio di Gemma inizia dai suoi cambiamenti, dalle sue sconfitte, disillusioni e ferite che lasciano indietro le aspirazioni negate, non ottenute, che portano alla maturazione. II viaggio, perciò, serve ad aiutarla ad accettare le parti dolorose per continuare con la vita, per respirare: "Avevo bisogno di amici e di ricordi. Non mi bastava la mia vita. Avevo bisogno di qualcuno che mi costringesse a soffrire, di un testimone, di uno che c'era". (Mazzantini 2010:488)

Nel romanzo si parla anche del viaggio di speranza per Pietro, figlio di Gemma, alla ricerca della propria origine a Sarajevo, "dove il passato pesa e fa rumore, come un barattolo al piede", ma anche per altri protagonisti, i quali ritornano lì dove il loro rapporto si è interrotto e dove ha il suo nuovo inizio, a Sarajevo.

Ma questo romanzo è anche un viaggio nel male della Storia con il quale si cerca di restituire la dignità e la forza al popolo di Sarajevo, in un periodo in cui l'oblio, il male e la sofferenza dominano nel mondo. Si tratta, quindi, di un "passaggio all'altra parte: la parte oscura del mondo" (Rella 2000:165), di un grido contro il silenzio, rivendicando così alla scrittura il diritto di parlare chiaramente delle verità scomode e delle malvagità del mondo con parole taglienti e precise, poiché "l'unica forma che sembra poter includere in sé questo limite [il male] è il racconto". (Rella 2002:15)

Però, questo romanzo non è solo la storia della guerra, è piuttosto la storia di piccole guerre quotidiane e le loro sconfitte, della ricerca di maternità. Si tratta, quindi, di un viaggio nel profondo dell'anima, cioè del viaggio verso il suo punto estremo. Secondo Rella, "è necessario procedere nel «cuore di tenebra» della passione, del pathos, dell'angoscia di fronte all'amore e alla morte, attraverso le lacerazioni, il male e il dolore per dare ad essi [...] la forma che li renda cosa nostra. Per fare questo è necessario spingersi [...] all'estremo". (Rella 2000:173) Questo viaggio nell'estremo, con la sua struttura complessa e minuziosamente progettata, si rivela come una navigazione traumatica attraverso un labirinto che si risolve soltanto alla fine, e la cui forza sta proprio nel fatto che lascia il lettore con un'infinita e indistruttibile «speranza di sperare».

\section{II dolore sdoppiato: la maternità negata e il male della Storia}

Venuto al mondo è il romanzo in cui si focalizza il "ventre": il ventre sterile di Gemma e quello sanguinante di Sarajevo, che si confondono in un'unica storia sul dolore. Quello che collega il ventre di Gemma e quello di Sarajevo è la ricerca della maternità, la sofferenza che avviene per la mancanza della realizzazione di essa, e la guerra a Sarajevo, grazie alla quale Gemma ottiene finalmente un bambino.

"Nel caso di Venuto al mondo la menomazione è quella della sterilità: una giovane donna che non riesce ad avere figli, e che rimane prigioniera di questa impossibilità; una coppia che insegue il sogno di un figlio come prova di esistenza, e che questa prova la trova in un percorso di iniziazione". ${ }^{14}$ Ma questo percorso simboleggia allo stesso tempo la ricerca della possibilità di ripartire per gli abitanti di una città, la ricerca della libertà; perciò la storia di Gemma si trasforma nel desiderio di un nuovo inizio per la città stessa.

Conseguentemente, Gemma si sente sempre "all'altezza di niente": "Sono malata d'incompletezza, di illusioni". (Mazzantini 2010:97) Gemma, quindi, si presenta come un'anima sofferente, nonostante in essa sia sempre presente una forte contestazione della sofferenza:

"In realtà l'unica cosa che so di me è che non mi piace soffrire" (Mazzantini 2010:46)

"Non volevo soffrire. [...] io non ero destinata a rincorrere chimere, a raccattare lacrime. II mondo mi sembrava saturo di tutto. Gli amori erano, come il resto, cancerosi di nostalgia ma svelti nel consumo. Era da fessi crederci. Tornavo a sentirmi in pace, benedetta dalla normalità, dal benessere moderato. (Mazzantini 2010:66)

Non sorprende che, dopo l'aborto, Gemma cerca di cancellare i ricordi di quanto successo e di staccarsi dal dolore, causato non solo dalla perdita del bambino, ma anche dalla possibilità mancata di creare una famiglia completa con Diego. Questo rifiuto del dolore nasce da un desiderio di mera sopravvivenza e protezione, dal disinteresse e dall'apatia del tempo contemporaneo, dalla paura di vivere in un mondo che ha rispetto solo per i piaceri, in cui lo scorrere del tempo non permette punti di sosta per la riflessione, per le domande che valgono la pena di essere chieste, pur essendo capaci di portare la sofferenza, che è la malattia più grande del secolo dalla quale si fugge invano come da una tempesta. Tutta la cultura dell'uomo è fondata sull'inimicizia verso qualsiasi esperienza del dolore. In questo tipo di società "il dolore appare privazione di un bene, frattura, segnale, appello: un ordine è interrotto, una armonia è infranta; e

14 Annunziata, L. Margaret va alla guerra. In La Stampa (http://www.lastampa.it/redazione/cmssezioni/cultura/200812articoli /39009girata.asp (11.12.2008.) (15.06.2012.)). 
la coscienza di questo disordine assume il volto di una maledizione e di una condanna". (Matteucci 1988:14)

Tutta la vita umana è la ricerca della felicità, o "di una base per la condizione di essere felici". (Frankl 1998:65) Proprio ciò manca a Gemma; un bambino con cui la sua felicità e quella di Diego sarebbe completa. Dopo le frustrazioni per le gravidanze interrotte, Gemma ottiene la diagnosi di "sterilità totale", con la quale comincia il periodo della sua profonda tristezza. II bambino diventa così una vera e propria ossessione di Gemma che la porta alla sconsolata conclusione: "Io non soffro più. Ho già sofferto. Forse mi sento addirittura sollevata. Non sarò mai una madre. Resterò per sempre una ragazza. Invecchierò così, asciutta e sola. II mio corpo non si sformerà, non si moltiplicherà. Non ci sarà Dio. Non ci sarà raccolto. Non ci sarà Natale. Invecchierò così. Morta, ecco come mi sento" (Mazzantini 2010:151), mentre il suo stesso nome sembra racchiudere la sua sofferenza, ovvero rimanda "all'incapacità di gemmare e procreare". 15

In questo modo, Gemma diventa un peso per se stessa: si sente lontana dalla felicità, dalla vita stessa, "una sorta di molecola, di atomo o di pianeta, di frammento totalmente separato dal resto delle cose, completamente concentrato su se stesso" (Argullol 2000:22): "Sono al chiuso del mio corpo piombato. II mondo si tenesse i suoi figli, io mi tengo la mia incompatibilità alla vita". (Mazzantini 2010:154) In altre parole, "dinanzi al dolore si può reagire rifugiandosi nell'isolamento, cadendo nell'autocommiserazione 0 in un atteggiamento di rivolta ostile contro il mondo e gli altri. È un comportamento comprensibile perché, in un certo senso, il dolore ci rende estranei [...], ci rende soli sia oggettivamente, poiché lo spazio del mondo per noi si restringe, sia soggettivamente". (Russo 2003:114) Nel caso di Gemma sono presenti entrambi i modi per affrontare il dolore. Per lei, il mondo è diviso in due: "Io faccio parte della metà livida. Come le foreste bruciate, i mari soffocati dalle alghe, le donne di Chernobyl. [...] Questa città mi sembra all'improvviso piena di donne incinte. Prima non ci facevo caso, adesso mi paiono un esercito. Un impavido battaglione che marcia contro di me." (Mazzantini 2010:152)

La maternità risulta per la protagonista un progetto destinato ad incontrare sempre gli ostacoli insormontabili e, alla fine, a fallire. L'unica speranza rimasta, dopo il tentativo fallito di adozione di un bambino, è l'affitto dell'utero, l'idea che la porta prima in Russia, dove con i soldi "si può fare tutto", e poi a Sarajevo, dove conosce Aska, una giovane donna disposta ad avere un figlio da Diego affinché questo atto la aiuti economicamente. La nascita di Pietro, attraverso la sofferenza di Gemma, rimanda così alla sofferenza di Cristo, dato che in tutti e due i casi il bene nasce dal male: dalla crocifissione, d'una parte, e dallo stupro, d'altra. La nascita di Pietro si intreccia, quindi, con la rinascita di Sarajevo, della Bosnia. Per questo motivo si può dire che il dolore si sdoppia: al livello della protagonista ed al livello dell'ambientazione.

La Bosnia occupa un posto importante nella vita di Gemma e di Diego, in quanto il loro primo incontro succede proprio a Sarajevo, dopo le Olimpiadi, e vi ha luogo anche il loro ultimo incontro, durante la guerra. Conseguentemente, nel corso del romanzo la vita serena degli abitanti di Sarajevo viene interrotta dalla violenza della guerra, anche se nessuno sapeva "che cosa abbia potuto trasformare una città serena e colta, multietnica e pluralista, fiera della sua modernità e del suo patrimonio di tradizioni in un luogo infernale"16, in un luogo della pulizia etnica. Si va così incontro alla descrizione del male che diventa il contagio e la contaminazione (Rella 2002:21), e dell'indifferenza umana, della coscienza vuota, che l'unica sembra poter spiegare tanta malvagità. Fare del male, infatti, significa ridurre l'uomo ad una cosa, "ridurre tutto allo stesso niente, un cesso pubblico e un convento nello stesso ammasso di calcinacci, un uomo morto accanto a un gatto morto". (Mazzantini 2010:209) Anche la Bosnia rappresenta un corpo, un corpo che incarna le sofferenze di tutti gli abitanti coinvolti in guerra.

\section{Il corpo come simbolo dell'identità e come metafora della città}

Di conseguenza si può dire che il romanzo Venuto al mondo è anche la storia del «ventre», dell'interno, dell'assenza di un corpo e della sua importanza.

II corpo ${ }^{17}$ si può percepire in diversi modi: come custode della mente, come corpo del dolore, della passione, della

15 Mondo, L. Margaret Mazzantini, "Un figlio «rubato» nell'inferno di Sarajevo". In La Stampa, dicembre 2008: http://margaretmazzantini.com/2008/12/un-figlio-\%C2\%ABrubato\%C2\%BB-nell\%E2\%80\%99inferno-di-sarajevo/(09.12.2008.)

(15.06.2012.)

${ }_{16}$ Casagrande, G. Venuto al mondo di Margaret Mazzantini. http://www.wuz.it/recensione-libro/2827/venuto-mondo-margaretmazzantini-sarajevo-guerra-bosnia.html (10.12.2008.) (15.06.2012.).

${ }^{17}$ Quanta sia l'importanza del corpo, in quanto inscindibile dall'anima, viene rilevato da Salvatore Natoli: "[...] la corporeità è l'esperienza costante del nostro essere noi stessi. In noi non c'è separazione tra corpo e mente, perché l'esperienza prima della mente è proprio il pensarsi come corpo. Quindi, non ci può essere una separazione tra anima e corpo;" (Natoli 2012:15). 
tortura, il corpo come parte dell'io, come oggetto di manipolazione, come mezzo di comunicazione. ${ }^{18}$ Natoli rileva che "la mente è [...] l'auto-riflessività del corpo su se stesso in quanto distinto dal suo ambiente" (Natoli 2012:18) dato che "la mente va oltre i limiti della pelle ma non va oltre i limiti del corpo, perché la memoria è sempre una memoria del corpo." (Natoli 2012:17) Quanto il corpo ci sfugge capiamo tenendo a mente che "non conosciamo mai le ragioni del corpo; ciononostante il corpo è l'attualità oggettiva sempre presente in base al quale sono costruite le relazioni." (Natoli 2012:20) Sapendo che "percepiamo sempre il nostro corpo come rapporto, non come cosa in sé" (Natoli 2012:21), la frustrazione di Gemma cresce sempre di più perché è proprio il suo corpo che le sfugga ma allo stesso tempo determina il corso della sua vita. Quindi, nel romanzo il corpo è fonte d'incompletezza e oggetto di distruzione, ma è anche la città stessa un corpo sofferente, che sfugge alla violenza e cerca di liberarsi dalla prigionia e di ripartire.

II corpo, quindi, si presenta come fonte di insoddisfazione, la forma in cui si vive catturati. Per Gemma il corpo è la sua carcere, il simbolo eterno dell'incompletezza perché le viene negata la possibilità di diventare madre. ${ }^{19}$ Così, nella descrizione della visita di una sua amica che ha partorito, Gemma sente una profonda disperazione. La sua afflizione è legata anche all'impossibilità di vivere la trasformazione del corpo legata al parto, la quale segna per sempre un corpo come il corpo nel quale il dolore ha procreato una vita ${ }^{20}$ :

Ogni volta che sono andata a trovare una mia amica che partoriva tra cuscini bianchi e fiocchi [...] mi sono sentita un pezzo più sola, un pezzo più brutta, [...] senza essere più io. Io non ho partorito. Non si guarisce mai da ciò che ci manca [...] lo non ho partecipato all'evento primigenio, alla rigenerazione di me stessa. [...] I parti cambiano le ossa, le spostano. Mia nonna diceva che ogni nascita è un chiodo nel corpo di una donna, un ferro di cavallo. E che prima di morire le madri rivedono i parti che hanno fatto, il corpo che si spalanca e cede al mondo carbone bianco. Vedono i chiodi, la traccia del loro percorso. Morendo cosa ricorderò? Quale sarà il mio ferro di cavallo? (Mazzantini 2010:390)

Gemma si ribella al suo dolore nato da questa «incompletezza» del corpo proprio attraverso le modificazioni artificiali del corpo stesso spostando l'attenzione da esso e non pensando all'impossibilità di avere figli. È una via di fuga per lei, dal momento che, ogni volta che si guarda nello specchio, vede il buco, le rughe che lasciano tracce delle sconfitte, la "pancia morta"21: "In bagno, mentre mi trascino via il trucco dagli occhi, mi guardo. Non mi piaccio. Vedo sempre e solo il buco mio". (Mazzantini 2010:328) Si sente già mutilata, priva di bellezza; sente anche la repulsione nei confronti del corpo. ${ }^{22}$ Perciò l'inizio della menopausa rappresenta la fine della sua rabbia: "Sono andata in menopausa un

${ }^{18}$ Sono numerosi gli esempi in Venuto al mondo che riguardano l'uso del corpo come mezzo di comunicazione: ad esempio, dopo la morte di Diego il corpo di Gemma si trasforma nel sorriso che si presenta come il segno dell'ultima disperazione, dell'ultima rivelazione dell'insensatezza della vita, rafforzata con l'anafora scoperta nella ripetizione della parola "sorrisi": "Sorrisi, era morto cadendo, accanto al mare come suo padre. Sorrisi perché non ero sorpresa. [...] Sorrisi perché non sapevo se sarei sopravvissuta, ma non mi preoccupavo". (Mazzantini, 2010:441.). D'altra parte, tutto il dolore imprigionato nel corpo di Armando, padre di Gemma, viene esposto, dopo la morte di Diego, nel suo aspetto esteriore, acquisendo la forma di una smorfia: "mi faceva impressione quella faccia storpida che non riconoscevo, la voce impastata, il torpore dell'unico occhio aperto. [...] La sua faccia sembra austera, perciò sorprende scoprirla così mobile. [...] dietro questa faccia miseramente storta c'è un segreto laido. Sembra contratta in un urlo fermo lungo un fiume, come in quel quadro dell'uomo solo con la sua bocca nera". (Mazzantini 2010:436-438.). Gli esempi del corpo come mezzo di comunicazione sono assai frequenti anche in altre opere della Mazzantini.

${ }^{19}$ Grazie alla tradizionale sottomissione della donna all'uomo e al secolare potere maschile, la donna ha imparato che la divisione dei ruoli concentra la sua funzione a quella dell'identificazione totale con il corpo. Ne risulta che, soltanto attraverso la maternità, la donna può avvicinarsi in parte al potere, quasi completamente riservato all'uomo, ed esercitare sul figlio, attraverso l'amore materno, una completa dipendenza del figlio del suo corpo. Questa forma di potere le permette di condizionare ed influenzare per sempre la vita del figlio, grazie al legame che si istaura tra il corpo del figlio e quello della madre.

${ }^{20}$ Quando nasce un figlio la donna è segnata per sempre dal dolore, il quale viene accentuato durante il parto, ma continua anche dopo, con le conseguenze del parto. Tuttavia, anche la donna che non ha figli soffre comunque perché il suo male emotivo per la mancata procreazione diventa una sofferenza fisica.

${ }^{21}$ Inoltre, il momento del parto rappresenta uno dei momenti più significativi per lo sviluppo della personalità, legato al distacco dalla figura materna. È anche un momento di liberazione, al quale Gemma non riesce ad arrivare: "Ė difficile non vedere analogie con l'atto di espulsione (o di estraniazione) con cui il soggetto storico ha creduto di poter rappresentare la sua nascita: congedo da una matrice limitante e mortale, divenuto poi modello per ogni liberazione che non riconosca delle necessità biologiche." (Melandri 2001:25).

22 || rapporto problematico con il proprio corpo è legato al fatto che Gemma sente che la sua vita possa avere un senso solo se un altro essere si distacca dal suo corpo, dato che questo garantisce la continuità dell'umanità. Però, paradossalmente, mentre perseguita tale obiettivo, dimostra di non essersi mai staccata dalla figura materna e rimane incastrata all'immagine della madre procreatrice: "[...] la storia appare attraversata da un movimento opposto: corsa verso il passato, «regressione fin dentro il ventre materno». Nostalgia e speranza coincidono: il paradiso a cui si mira è lo stesso da cui siamo partiti." (Melandri 2001:21). 
anno fa, non me ne è fregato un accidenti, non mi è mai servito a nulla il mio ciclo. La fine del sangue è stata la fine della rabbia verso me stessa". (Mazzantini 2010:489)

D'altra parte, attraverso il caso di Aska, la madre naturale del figlio adottato da Gemma, viene rappresentata la distruzione del corpo, in quanto sul suo corpo viene eseguito il peggiore tipo di violenza, lo stupro, un atto inscindibile dalla guerra in Bosnia, il "tentativo sistematico di minare l'identità femminile". (Di Palma 2010:224) Gli stupri delle donne in guerra sono collegati alla forza con la quale l'uomo sostituisce la propria paura e invidia nei confronti della donna, in quanto, a differenza dell'uomo, ha la possibilità di procreare. Questa caratteristica esclusivamente femminile incute paura all'uomo perché sottolinea la sua vacuità, così che l'uomo, conseguentemente, cerca di sottoporre la donna al proprio dominio attraverso lo stupro. ${ }^{23}$ Lo stupro si rivela, quindi, uno dei metodi per dominare la donna, dato che l'uomo si sente minacciato da questa specificità femminile, in quanto interiore, biologica e intoccabile. ${ }^{24}$ Dunque, attraverso lo stupro l'uomo esercita il suo potere sulla donna per riconfermare la propria forza e superiorità. Detto altrimenti, attraverso lo stupro l'uomo esercita il controllo sulla donna. II potere di partorire, che provoca paura nell'uomo in quanto sfugge al suo controllo, diventa così controllato almeno in parte, fecondando "i campi di semi cattivi", usando, cioè, la forza e il dono regalato alla donna come l'arma contro di essa, contro la sua identità.

Tuttavia lo stupro rileva anche che il corpo non è qualcosa di cui l'uomo dispone. (Zlatar 2010:107) Dopo lo stupro, Aska non può non sentire il corpo; se riusciva a farlo grazie alla sua immaginazione, adesso, comunque, esso non le sembra possibile perché il bambino vive dentro di lei: "il catarro dei diavoli", "il coagulo dei diavoli", "il nido dei serpenti", l'orrore interno che la ricorda che non c'è il limite tra l'interno e l'esterno, che la pelle non può proteggerla dal mondo esteriore. Per questo motivo lei non può rientrare nel suo corpo, dal momento che esso è già "abitato". Ed è la memoria

${ }^{23}$ L'incomprensione del ciclo mestruale da parte degli uomini si può notare anche in un altro personaggio della Mazzantini, Timoteo in Non ti muovere, nell'occasione quando sua figlia affronta la prima mestruazione che consolida il rapporto della ragazza con la madre, mentre esclude la presenza del padre: "Mi sono ritirato qua dentro, a voi donne il resto della casa, panni bianchi, ovatta, sangue di vergine. Tua madre ha preparato il tè [...] Inzupperete biscotti con le gambe incrociate sul tappeto come due coetanee. Oggi è una giornata speciale [...]. lo mangerò un po' di formaggio da solo in cucina, più tardi. Penso che un giorno farai l'amore". (Mazzantini 2001:128).

Riguardo il ciclo mestruale, è importante sottolineare la paura del sangue nell'uomo, visibile in Timoteo che ha paura del sangue: "[...] l'incisione è l'unico momento che mi provoca una piccola vertigine". (Mazzantini 2001: 47). Nell'occasione in cui viene costretto ad operare la sua amata Italia, lui capisce che questo timore è dovuto alla paura del sangue di Italia, della quale solo adesso diventa cosciente: "Avevo già vissuto quel momento, e forse lo avevo atteso. Avevamo quell'appuntamento. E mi sembrò di penetrare finalmente la mia vita. La paura del sangue che avevo da ragazzo, l'incisione, quell'attimo bianco in cui la carne già incisa non sanguina ancora... forse era lei. Lei era in quel taglio. II sangue che temevo era il suo, così come avevo temuto il suo amore". (Mazzantini 2001:264.). Ciò si rivela importante perché la paura del sangue e dell'amore è connessa alla paura ed al rifiuto della madre e, conseguentemente, del femminile, il che ha come risultato lo stupro della donna amata dalla parte di Timoteo. Detto altrimenti, l'ordine sociale e la cultura negano l'importanza dell'utero, cioè, della prima dimora e del primo amore dell'uomo, in quanto la repressione riguardo alla primaria dipendenza del bambino dalla madre, riguardo alla necessità ed al desiderio i quali il corpo della madre suscita nel bambino dopo la nascita, sono necessari per stabilire l'autonomia e il predominio dell'uomo. (Cfr. Irigaray 2000:418.). Per questo motivo, Irigaray, inoltre, scrive che l'utero, essendo negato nella cultura, viene visto da molti uomini come un pericolo per la loro supremazia ed autorità. In più, a causa delle rappresentazioni mancanti della sessualità femminile, l'immagine dell'utero si confonde con il sesso femminile, il quale provoca l'ansia, la paura, la ripugnanza, per cui risulta che esso deve essere sottomesso al dominio maschile (cfr. ibid.), cioè, disprezzato, il che trova la sua peggiore conseguenza in questo romanzo nello stupro. In tal modo si cerca, quindi, di dimenticare la dipendenza dalla madre e di distruggere il potere materno e femminile. Esso, tuttavia, determina la vita dell'uomo. In questo senso, bisogna anche menzionare il desiderio conseguente di Timoteo di "soggiornare" nel ventre di Italia, il quale nasce in lui dopo gli stupri iniziali, cioè, dopo la paura iniziale della donna: "Non avevo più paura di lei, le pesavo addosso come un uomo, come un figlio". (Mazzantini 2001:106.). Ciò può essere interpretato come la ricerca di un rifugio nel corpo, un nuovo incontro con la prima dimora dell'uomo, con la sicurezza di un bambino. Esso, quindi, rappresenta la fuga da un mondo adulto, da una vita fallita, da una paura di vivere, nonché la voglia di una rinascita, di un nuovo inizio che includerebbe il rispetto verso la madre, "la madre in ogni donna e la donna in ogni madre" (Cfr. Irigaray 2000:420.): "Baciai l'ombelico di Italia. [...] Ora mi sembrava di poterlo penetrare, di poter schiudere con le labbra quell'uscio molle per infilarci dentro [...] tutto me stesso. Sì, volevo essere nel suo ventre, [...]. Chiusi gli occhi nella mia saliva. Ero un neonato nel suo fondo d'acqua. Fammi nascere, fammi rinascere, amore mio. Avrò più cura di me stesso, ti amerò senza maltrattarti". (Mazzantini 2001:156.).

${ }^{24}$ Andrea Zlatar, ad esempio, nell'analisi del tocco riporta l'idea di Julia Kristeva sull'ordine patriarcale nel quale il potere maschile conferma di essere minacciato dalla forza irrazionale e furba che esso non può controllare, e che si riferisce alla forza del pericolo rappresentato dal sangue mestruale. Questa forza proviene dal fatto che viene dal dentro, dalla parte interna dell'identità sociale 0 sessuale, il che minaccia la base di rapporti sociali tra i sessi. (Cfr. Zlatar 2010:26-27). 
della sua vergogna, della distruzione della sua identità. ${ }^{25}$

Inoltre, non è solo il bambino che la ricorda della violenza subita, ma anche il segno sulla nuca, il segno lasciatole dallo stupratore durante l'atto di violenza, che Diego trasforma in un tatuaggio, in una "cosa bella", per almeno cercare di allontanare il male, di seppellirlo. Non conoscendo la verità Gemma riconosce in questo tatuaggio "una tristezza tangibile", dicendo: "Mi sembra che in questa macchia rossa ci sia più guerra che in tutte le altre foto di guerra". (Mazzantini 2010:107) Detto altrimenti, il corpo di Aska si trasforma nel simbolo dell'intera Bosnia: esso simboleggia il corpo distrutto di tutta la gente di Sarajevo ("ha la Bosnia intera negli occhi"), e il suo ventre rappresenta il ventre di Sarajevo, violentato, massacrato ed insanguinato.

Ci sono molti esempi nel testo in cui la città stessa è rappresentata come il corpo, cioè, come un organismo che raccoglie in sé i pensieri ed i dolori di tutta la gente che vive in essa e che "può andare via come ogni cosa viva", destinata alla morte. Perciò a Gemma sembra che Sarajevo possa perfino "sussurrare" a Pietro, per cui ha paura che "Sarajevo abbia una voce sottile, che canti e racconti". Del resto, descrivendo la città con le metafore contenenti le parti del corpo (Miljacka sembra "latte di donna", Holiday Inn è un "ventre scuro", l'aeroporto è "un piede in cancrena in fondo al corpo sofferente della città", il Ponte Vecchio è lo "scheletro bianco" perché gli manca quel corpo che aveva prima della guerra), si allude alla fragilità della città, alla facilità con la quale può essere distrutta, proprio come il corpo umano. Oltracciò, ricorrendo alla metafora del corpo per rappresentare la città e le sue trasformazioni, si consolida ancora di più il tema centrale del corpo, cioè del ventre, nonché la simbologia che Aska e Pietro ottengono nel romanzo: la forza e la resistenza di Aska coincide con la difesa resistente di Sarajevo. In altre parole, il corpo di Aska resiste, come resiste il popolo di Sarajevo, dimostrando di essere più forte delle tragedie subite e dei tentativi disumani di distruggere la vita; di conseguenza, con la nascita di Pietro si allude alla futura rinascita della Bosnia e alla speranza che il male, alla fine, non sempre deve vincere.

\section{Mare al mattino: due tragedie incrociate dalla storia}

Mare al mattino è una parabola etica, un libro di argomenti politici sull'esclusione dell'altro, che attraverso il raddoppiamento dei dolori e del dramma delle due madri, due figli e due mondi, rappresenta come in uno specchio i destini incrociati di due paesi, l'Italia e la Libia, nella lotta per il dominio. Vi sono descritti due movimenti e una sola direzione (scappare dal deserto per attraversare il mare dalla Libia all'talia), e due periodi26, ma nessun vincitore, in un movimento globale circolare causato dall'arroganza del potere, in quanto "la storia è un millepiedi e ogni piede tira da una parte diversa, e in mezzo c'è il corpo nostro". (Mazzantini 2011:82) Nell'analisi del romanzo si può, quindi, parlare del doppio al livello della storia, al livello dei personaggi, ed al livello della natura.

In primo luogo il romanzo parla delle vittime di ieri e di oggi. Da una parte, si descrive la storia degli italiani espulsi, puniti per "le malefatte del colonialismo cruento e velleitario dell'Italia liberale di Giolitti e della quarta sponda fascista" (Mazzantini 2011:47), gli italiani come Angelina e la sua famiglia che in Libia hanno trovato la casa ("Fecero amicizia con gli arabi. [...] Erano poveri con altri poveri" (Mazzantini 2011:39-40)). D'altra parte, si rappresenta il destino tragico degli indigeni della Libia costretti a scappare dal regime, come Jamila e Farid, i quali Gheddafi lasciava fuggire dal proprio paese natio verso il Mediterraneo per far tremare l'Europa ${ }^{27}$. Nel romanzo, quindi, si problematizza come il progresso di diversi paesi, dovuto nel caso della Libia alla scoperta del petrolio, risulta con il controllo del resto del mondo: "non ti fidare di quello che sembra una fortuna. E sempre quello che per i ricchi è una fortuna, per i poveri è una disgrazia". (Mazzantini 2011:29-30) II progresso dell'uomo porta con sé inevitabilmente anche il dolore ed il male, mentre le conseguenze che ne risultano sono notevoli, visto che la sofferenza, dovuta agli spostamenti globali a causa dell'esercitazione del potere, affonda il mondo e rende gli uomini "muti, afasici, senza linguaggio come meri oggetti, relitti abbandonati su una squallida riva". (Rella 2002:9) Ciò diventa visibile soprattutto nel personaggio di Angelina la quale,

\footnotetext{
${ }^{25}$ È perfino difficile essere guardate da altre donne dopo lo stupro, e ancor meno mantenere il bambino, il frutto del male. Persino lo sguardo delle altre ricorda la donna le violenze subite sul proprio corpo; lo sguardo, così, si trasforma in un altro tipo di violenza. Ma guardare rappresenta il dolore anche per chi guarda, "gli occhi ammalano il corpo perché sono costretti a guardare". Anche Aska si rifiuta di guardare Diego poiché si vergogna del proprio corpo: "Diego le tiene la testa, lei non può guardarlo in faccia, non vuole. Si vergogna di se stessa, di quello che le hanno fatto". (Mazzantini 2010:511).

${ }^{26}$ Cfr. Valerio, C. Quelle donne fuggite in ciabatte. In La Stampa, dicembre 2011: http://margaretmazzantini.com/2011/12/quelle-donnefuggite-in-ciabatte (07.12.2011.) (20.06.2012.)

27 "Ė l'arma migliore che ha. La carne marcia dei poveri. È dinamite. Fa scoppiare i centri d'accoglienza, le ipocrisie dei governanti." (Mazzantini 2011:23.).
} 
costretta a lasciare Libia, da quel momento in poi vive proprio a metà, in solitudine anche in mezzo agli altri, come "la coda sporca di una storia coloniale che nessuno aveva voglia di dissotterrare". (Mazzantini 2011:68)

Detto altrimenti, attraverso la sua storia e la storia di Jamila la Mazzantini affronta il tema del postcolonialismo, dell'estraneità e della cattiva accoglienza degli italiani in Libia e degli africani in Italia. Questo argomento porta alla conclusione paradossale che nel mondo odierno, in cui regna la globalizzazione, risulta tuttavia difficile accettare e rispettare una cultura diversa dalla propria. Considerati sempre gli "altri", vivendo tra le due culture e le due nazioni, tali individui rimangono in una terra di mezzo, non appartenendo più a nessuna, costretti alla vita ai confini ed all'identità dei profughi: "Noi siamo tripolini, non siamo né qui né lì, siamo fermi in mare come quei ragazzi senza approdo". (Mazzantini 2011:115) La famiglia di Angelina sente perfino la colpa a loro attribuita dopo il ritorno in Italia ("Cosa siete tornati a fare? A rubare il lavoro agli altri italiani, quelli veri, nati e cresciuti qui?" (Mazzantini 2011:69)), facendo così nascere un mondo quasi kafkiano in cui la condanna non avviene dopo il crimine ma in cui proprio la punizione imposta ad un individuo miserabile lo costringe a cercare il delitto di cui è accusato, cioè la sua colpa:

Santa smise di lottare. Da qualche parte cominciò a sentirsi colpevole. A sembrarlo. Non riusciva a staccarselo di dosso quel sentimento di smarrimento, di minorità. La gente privata di se stessa perde i confini, messa al muro può confessare un omicidio che non ha commesso. (Mazzantini 2011:69)

Conseguentemente, Angelina, sentendosi smarrita, senza una vera casa, è sempre pronta a ripartire, e pensa solo di "Riportare la sua vita [...] Nel punto dove si era interrotta". (Mazzantini 2011:74)Comunque, dopo il ritorno in Libia, Angelina capisce che quello non è più il suo paese, in quanto Tripoli è distrutta, ed esiste solo la memoria di quello che era stata una volta. Perciò ride "come una demente", godendo la mutilazione e la trasformazione della città che in tal modo non la fa più soffrire tanto come prima, ma la libera della sua disperazione, del male interno. Libia ora ricorda il Ground Zero, come sottolinea Vito, figlio di Angelina, un ragazzo combattuto tra la spensieratezza adolescente e l'assunzione delle responsabilità delle proprie azioni e delle decisioni che dovrebbero contribuire alla creazione di un mondo migliore. Di conseguenza, il viaggio in Libia si dimostra utile non solo per Angelina ma anche per Vito, che ha vissuto con la madre per diciotto anni senza mai capirla veramente, senza capire come la storia crudele del mondo possa negare alle sue vittime la felicità e la pace. II viaggio, quindi, risulta importante per la relazione tra la madre e il figlio: d'una parte, il ritorno nello spazio familiare è per Angelina il ritorno nel passato, la consapevolezza che concerne l'impossibilità di continuare la vita là dove si era interrotta, il confronto con se stessa, ossia con quello che è diventata in "esilio", mentre, d'altra, ciò aiuta anche a Vito a capire la madre per la prima volta, il suo mal d'Africa che l'ha marcata:

Quel male minore, caduco, fatto di attacchi che poi se ne vanno come febbri malariche. E dopo restano quegli occhi di vetro feriti, la lingua dolorante, che non può parlare. Come se fosse stata morsa da dentro, da un animale nascosto. Adesso l'animale era uscito, sontuoso, vorace. Vito guardò la madre muovere i fianchi e la pancia in un altro modo, come avesse preso il battito di quel mare li, di quelle onde lunghe [...]. Aveva fatto la biopsia della città. Aveva analizzato le cose cattive che avevano rimpiazzato le cose belle scomparse, e adesso si godeva quella mutilazione. (Mazzantini 2011:91-92)

D'altra parte, a differenza di Angelina, Jamila e altri profughi cercano soltanto di non guardare indietro, vogliono sperare che dopo il mare c'è la salvezza, l'aiuto. ${ }^{28}$ Più precisamente, la vita di tutte e due le protagoniste è altrove, è una vita rimandata nel tempo e nello spazio. Oltre alle due madri, anche i due ragazzi si differenziano uno dall'altro: mentre Farid è ancora un bambino che cerca il rifugio nella madre e segue i passi di lei, costretto a vivere la trauma della morte del padre e della fuga dal paese natio, Vito è quasi un uomo che deve accettare la responsabilità della propria vita e agire, portando cambiamento. II suo tentativo di trovare un luogo che gli corrisponda, di creare un sistema interiore di valori, è in conflitto con il mondo esteriore che ha la forza di distruggere la vita. Vito capisce che vive in un mondo di odio e di dolore, incapace di non ripetere gli errori del passato. Dopo la seconda guerra mondiale, diversi popoli sono coinvolti nelle guerre locali le quali, tuttavia, riguardano l'intero mondo. Vito capisce questa universalità del dolore che unisce tutti nella stessa tragedia; lui vede nella vita della madre quanto possano essere durevoli le conseguenze di una volontà irragionevole del potere e quanto possano cambiare la vita. Di conseguenza, Vito comprende che gli orrori si dimenticano

${ }^{28}$ La Mazzantini descrive l'accoglienza degli africani in Italia in questo modo: "Ha visto la saturazione, la paura delle epidemie. La gente protestare, bloccare i moli, gli approdi. E poi ricominciare, buttarsi nel mare in piena notte per tirare su quei disperati che nemmeno sanno nuotare". (Mazzantini 2011:113). La scrittrice rappresenta così l'ambiguità ed incertezza che riguarda l'aiuto agli sventurati da parte di quelli che sono più fortunati, che scatta per la paura dello "sconosciuto". 
e si nascondono, ma che dovrebbero essere conservati almeno nella memoria, per ricordare cosa possa causare l'odio. Perciò in lui nasce il desiderio di cambiare il mondo:

Ė quello che un ragazzo dovrebbe sperare, partecipare all'organizzazione del mondo. Lui è sempre stato uno studente in fuga, e non solo dalla scuola. Da ogni forma di apprendimento. Abbassa la testa. Si vergogna di queste ambizioni improvvise. Non farà niente di buono, di rimarchevole. Ė più facile che accada così, che la sua vita passi inosservata. (Mazzantini 2011:76)

Eppure, quello che collega la sua storia a quella di Farid, oltre all'amuleto che Vito trova in spiaggia, è proprio la mancanza di un futuro migliore. E mentre per Farid e Jamila non c'è nemmeno speranza di futuro29; il futuro è negato anche a Vito ed Angelina, visto che esiste solo la violenza che paradossalmente crea la libertà. Perciò la fine del libro non è una fine felice ma amara ed ironica: "Evviva evviva", perché la morte di Gheddafi, "L'insensatezza della rabbia postuma", "un macabro trofeo [...] sporca i vivi". (Mazzantini 2011:123) Risulta che alla violenza si reagisce con la violenza, e l'odio genera l'odio. Nel momento stesso in cui si sottrae umanità alla vittima si sottrae umanità a se stessi. (Rella 2002:136) In questo senso, quando viene ucciso un uomo, anche se un violentatore, tiranno, assassino, non inizia il cammino verso la pace ma verso una più forte presenza del male e di una triste vittoria.

In ultimis, le due storie sdoppiate rimandano anche allo sdoppiamento nella natura, ovvero nel deserto, un "deserto di sabbia, e di mare, che inghiotte i destini e ogni tanto rende qualcosa" ${ }^{30}$, che rivela così la forza dominatrice della natura. D'una parte c'è il deserto che "non si rivela mai, appare e scompare. Ha un volto che cambia forma e colore, vulcanico o bianco di sale. Un orizzonte invisibile, che danza e si sposta come le sue dune" (Mazzantini 2011:16); e d'altra, si trova il mare, "monotono, non ha nessuna novità. Guardarlo è uno sbaglio, è come guardare un animale senza testa. [...] Carne blu che schiuma da una bocca sommersa. Farid [...] Si chiede qual è la faccia del mare". (Mazzantini 2011:27) II deserto ed il mare rappresentano tutti e due il pericolo, in quanto hanno una logica e una forza superiori all'uomo ed alla sua fragilità. Ciò si capisce soprattutto nella rappresentazione del mare, la cui importanza è accennata già nel titolo, il quale viene mutato nel romanzo in un'anima pulsante, simbolo della libertà e speranza, ma anche della prigione, di una liquida tomba, la cui forza viene accentuata dal linguaggio visionario della scrittrice. ${ }^{31}$

In questo modo il mare diventa il ponte ed il confine che unisce i due paesi in un libro di tristezza, nostalgia e disperazione, ma anche di coraggio, del tentativo di ricominciare. D'altra parte, la fuga, il viaggio attraverso il mare, verso lo sconosciuto, influenza anche la trama, che è molto veloce, in quanto l'autrice riesce a rappresentare in poche parole $\mathrm{e}$ soprattutto attraverso le enumerazioni la densità delle emozioni e degli eventi, nello stesso modo in cui è in grado di descrivere il mondo di due famiglie per mettere allo scoperto la storia dimenticata di intere generazioni. In questo senso, invece della ricerca individuale del passato per vincere l'oblio, la Mazzantini trasforma lo scopo nel ruolo collettivo che riguarda lo scavare degli eventi della storia, dal momento che la cancellazione dei ricordi dolorosi e la dimenticanza delle colpe non può portare nessun cambiamento: "Sotto il piede di ogni civiltà occidentale c'è una piaga, una colpa collettiva. La madre non ama chi si professa innocente. [...] Angelina dice che lei non è innocente. Dice che nessun popolo che ha colonizzato un altro popolo è innocente". (Mazzantini 2011:79)

Quindi, occorre sottolineare che in Mare al mattino la Mazzantini riesce a cogliere l'immagine di una realtà frenetica, frammentaria ed inconcludente, in quanto dimostra il fenomeno antropologico del non-cambiamento e dell'irresponsabilità, nonché la ripetizione storica dell'ostilità, della crudeltà insensata del regime totalitario, la colpa collettiva del mondo, la vita degli «altrì» e la marginalità di essi, la necessità di dare appoggio e di aiutare quelli che ne hanno bisogno. In questo senso si può dire che le storie dei due figli, delle due madri e dei due mondi, si specchiano e, alla fine, disegnando una sola storia, quella dell'intera umanità, che deve imparare a rispettarsi e convivere, diventando così "quello che la vita dovrebbe essere: esperienza che si moltiplica, cure che trovano altre cure, pensieri che incontrano

${ }^{29}$ La Mazzantini usa il futuro come tempo verbale per parlare dei progetti e dei sogni di Farid e Jamila che al lettore risultano tragicamente ironici in quanto impossibili: "Farid aspetta l'alba. [...] Gli daranno dei giocattoli, gli daranno la coca-cola e la pizza". (p. 30), nonché per accennare in questo modo alla loro morte: "Nessuno approderà da quella barca. [...] Una nave passerà lontana senza fermarsi". (Mazzantini 2011:112.).

${ }^{30}$ Platzer, T. II nostro amore Venuto al mondo al Carignano. In La Stampa, novembre 2011: http://margaretmazzantini.com/2011/11/ilnostro-amore-venuto-al-mondo-al-carignano (23.11.2011.) (20.06.2012.).

31 "Il mare ormai è una miniera chiusa sulle loro teste, la casa del diavolo. [...] Ora aspetta soltanto il destino. L'ultima faccia della storia. La scruta, la cerca, la carne scavata dagli schizzi di sale, in un luogo dove non c'è più orizzonte. C'è solo mare. II mare della salvezza che adesso è un cerchio di fuoco bagnato. Un cuore nero". (Mazzantini 2011:105-106.). 
altri pensieri. Rispetto di se stessa, dell'ignoto che abita, dell'ignoto da cui è abitata". ${ }^{32}$

\section{Conclusione}

L'importanza dei romanzi analizzati è racchiusa nella capacità della Mazzantini di smascherare i temi come dolore, morte, odio, guerra, violenza, ma anche di affrontare le questioni come la solitudine, l'incomprensione, la paura di accettare la responsabilità, l'egoismo e l'incapacità di accogliere l'«altro» come parte dello stesso universo. Ciò che la scrittrice focalizza è il dolore dell'anima, il quale si combatte mentre si cerca costantemente di trovare la pace in un mondo nel quale i personaggi sono costretti a lottare incessantemente con la difficoltà di esistere. "Io sento gracidare il dolore del mondo", dichiara la Mazzantini33, cioè sente quel dolore che cerca di raccontare con lo scopo di denudare la sofferenza umana. Si può concludere che proprio grazie ai personaggi della Mazzantini si contribuisce al risarcimento della sofferenza e ad una più forte consapevolezza dell'ineluttabilità e dell'universalità del dolore.

Dall'analisi risulta che l'autrice riesce ad interpretare le crisi del suo tempo rappresentando la storia umana attraverso la descrizione del destino di personaggi che non sono degli eroi, ma i quali non si risparmiano nella loro ricerca di una vita migliore, nel rimorso, nella lotta contro l'insensatezza del mondo e nell'amore. Attraverso i suoi personaggi, i quali seguono il proprio codice etico e, conseguentemente, si autocondannano per le decisioni prese, la Mazzantini cerca di contribuire ad una più forte consapevolezza dell'ineluttabilità e dell'universalità del dolore. Utilizzando lo spazio come simbolo del dolore universale la Mazzantini trasforma il dolore individuale nel comune e lo rende universale. Questa universalità della sofferenza attenua la sofferenza della protagonista e trasforma il suo estraniamento, il suo sentirsi esclusa dal mondo in quanto sofferente, in un viaggio della speranza, ovvero in un racconto di un possibile nuovo inizio.

\section{References}

Agrò, F. E. (2003). La malattia e la sofferenza dell'uomo, in quanto unità somatico-spirituale: inquadramento anatomo-fisiologico ed approccio clinico. In Eslanda, R. \& Russo, F. (a cura di) Homo patiens. Prospettive sulla sofferenza umana (pp. 15-67). Roma: Armando.

Argullol, R. (2000). Davalú. Cronaca di un duello. Frammenti. In Rella F. (a cura di), Pathos: scrittura del corpo, della passione, del dolore (pp. 11-27). Bologna: Pendragon.

Bonelli, J. (2003). Il senso della sofferenza. In Eslanda, R. \& Russo, F. (a cura di) Homo patiens. Prospettive sulla sofferenza umana (pp. 68-93). Roma: Armando.

Carson, f. (2006). Feminism and the body. In Gamble, S. (edited by), The Routledge Companion to Feminism and Postfeminism (pp.94102). London: Routledge.

Di Palma, S. V. (2010). Lo stupro come arma contro le donne: l'ex Jugoslavia, il Rwanda e l'area dei Grandi Laghi africani. In Flores, M. (a cura di), Stupri di guerra. La violenza di massa contro le donne nel '900 (pp.216-224). Milano: Franco Angeli.

Dostoevskij, F. M. (1989). Memorie dal sottosuolo. Milano: Mondadori.

Frankl, V. (1998). Patnja zbog besmislena života, (titolo originale Das Leiden am Sinnlosen Leben, traduzione di Božidar Brezinščak Bagola). Đakovo: UPT.

Givone, S. (2011). Storia del nulla. Roma-Bari: Gius. Laterza \& Figli.

Greco, A. (2004). Fenomenologia del dolore: Riflessione teologica sul valore salvifico della sofferenza. Roma: Armando.

Irigaray, L. (2000). The bodily encounter with the mother. In Lodge, D. \& Wood, N. (edited by), Modern Criticism and Theory. A Reader (pp. 413-423). London: Pearson.

Kristeva, J. (1989). Moći užasa, (titolo originale Pouvoirs de l'horreur, traduzione di Divina Marion). Zagreb: Naprijed.

Leopardi, G. (1983). Zibaldone di pensieri. vol. I. Milano: Mondadori.

Mazzantini, M. (2001). Non ti muovere. Milano: Mondadori.

Mazzantini, M. (2010). Venuto al mondo. Milano, Mondadori.

Mazzantini, M. (2011). Mare al mattino. Torino: Einaudi.

Matteucci, B. (1988). Lettere sul dolore. Firenze: Fiorentina.

Melandri, L. (2001). Le passioni del corpo. La vicenda dei sessi tra origine e storia. Torino: Bollati Boringhieri.

Natoli, S. (2002). L'esperienza del dolore. Le forme del patire nella cultura occidentale. Milano: Feltrinelli.

Natoli, S. (2012). La verità del corpo. Milano: Edizioni Albo Versorio.

32 Mazzantini, M. Schegge di dolore. In La Repubblica, settembre 2002: http://www.repubblica.it/speciale/2002/11settembre Iscrittori/mazzantini.html (11.09.2002.) (20.06.2012.).

${ }^{33}$ Cfr. Brocardo, E. Questo libro ti mette incinta. In Vanity fair, maggio 2011: http://margaretmazzantini.com/2011/05/questo-libro-timette-incinta (18.05.2011.) (15.06.2012.). 
Pareyson, L. (1989). Filosofia della libertà. Genova: Il Melangolo.

Rella, F. (2000). Di fronte all'indicibile. In Rella, F. (a cura di), Pathos: scrittura del corpo, della passione, del dolore (pp. 163-175). Bologna: Pendragon.

Rella, F. (2002). Figure del male. Milano: Feltrinelli.

Rella, F. (2012). Ai confini del corpo. Milano: Garzanti.

Russo, F. (2003). II dolore: autointerrogazione ed esperienza della prova. In Eslanda, R. \& Russo, F. (a cura di) Homo patiens. Prospettive sulla sofferenza umana (pp. 99-116). Roma: Armando.

Verri, P. (1964). Discorso sull'indole del piacere e del dolore. Milano: Feltrinelli.

Zlatar, A. (2010). Rječnik tijela. Zagreb: Ljevak.

Annunziata, L. Margaret va alla guerra. In La Stampa (http://www.lastampa.it/redazione/cmssezioni/cultura/200812articoli /39009girata.asp (11.12.2008.) (15.06.2012.)).

Brocardo, E. Questo libro ti mette incinta. In Vanity fair, maggio 2011: http://margaretmazzantini.com/2011/05/questo-libro-ti-metteincinta (18.05.2011.) (15.06.2012.).

Casagrande, G. Venuto al mondo di Margaret Mazzantini. http://www.wuz.it/recensione-libro/2827/venuto-mondo-margaret-mazzantinisarajevo-guerra-bosnia.html (10.12.2008.) (15.06.2012.).

De Maglie, D. Dal Catino di zinco a Zorro: L'identità femminile in Margaret Mazzantini. http://www.altoadigecultura.org/pdf/r04_05.html (24.09.2011.) (15.05.2012.).

Mazzantini, M. Schegge di dolore. In La Repubblica, settembre 2002: http://www.repubblica.it/speciale/2002/11settembre/scrittori Imazzantini.html (11.09.2002.) (20.06.2012.).

Mondo, L. Margaret Mazzantini. "Un figlio «rubato» nell'inferno di Sarajevo". In La Stampa, dicembre 2008: http://margaretmazzantini.com/2008/12/un-figlio-\%C2\%ABrubato\%C2\%BB-nell\%E2\%80\%99inferno-di-sarajevo/ (09.12.2008.) (15.06.2012.).

Platzer, T. II nostro amore Venuto al mondo al Carignano. In La Stampa, novembre 2011: http://margaretmazzantini.com/2011/11/ilnostro-amore-venuto-al-mondo-al-carignano (23.11.2011.) (20.06.2012.).

Valerio, C. Quelle donne fuggite in ciabatte. In La Stampa, dicembre 2011: http://margaretmazzantini.com/2011/12/quelle-donne-fuggitein-ciabatte (07.12.2011.) (20.06.2012.). 